Cite this: Phys. Chem Chem Phys, 2013, 15, 9531

Received 23rd January 2013, Accepted 16th April 2013

DOI: $10.1039 / \mathrm{c} 3 \mathrm{cp} 50330 \mathrm{e}$

www.rsc.org/pccp

\section{Dependence on the structure and surface polarity of ZnS photocatalytic activities of water splitting: first-principles calculations $\dagger$}

\author{
Xiangying Meng, ${ }^{\text {ab }}$ Hai Xiao, ${ }^{b}$ Xiaohong Wen, ${ }^{a}$ William A. Goddard III, ${ }^{\text {b Song Li }}$ \\ and Gaowu Qin*a
}

It has been reported that phase structure and surface polarity largely affect the photocatalytic efficiency of semiconductor nanostructures. To understand the chemical activity of ZnS at the electronic level, we investigate electron structures and carrier transportation ability for bulk intrinsic zinc blende (ZB) and wurtzite (WZ) ZnS, as well as the reaction pathway of hydrogen generation from water splitting on $\mathrm{Zn}$ - and S-terminated polar surfaces. The electron structure calculations prove that the WZ phase possesses a higher reducing ability than the ZB phase. The conductivity of the bulk ZB phase surpasses that of the WZ phase at or above room temperature. As the temperature increases, the asymptotic conductivity ratio of WZ/ZB is close to the Golden Ratio, 0.62 . Reaction kinetics studies indicate that Zn-terminated polar surfaces are more chemically active than S-terminated polar surfaces in the reaction of hydrogen generation from water splitting. The calculation results suggest that the first $\mathrm{H}$ splitting from water on Zn-terminated polar surfaces can occur with ground state electronic structures, while photoassistance is necessary for the first $\mathrm{H}$ splitting on the $\mathrm{S}$-terminated surfaces. Electronic triplet states calculations further show that Zn-terminated surfaces are more photosensitive than S-terminated surfaces.

\section{Introduction}

Semiconductors have been investigated as photocatalyst materials for the technological solution to environmental pollution and energy shortage. ${ }^{1,2}$ As an important II-VI semiconductor with a wide direct bandgap ( $3.72 \mathrm{eV}$ for the cubic zinc blende (ZB) phase $^{3}$ and $3.77 \mathrm{eV}$ for the hexagonal wurtzite (WZ) phase ${ }^{4}$ ), zinc sulfide ( $\mathrm{ZnS}$ ) has highly negative conduction band levels, ${ }^{5}$ thus has a higher theoretical efficiency of photocarrier generation and stronger reducing power than $\mathrm{TiO}_{2}$, which could result in a higher photocatalytic efficiency of ZnS. In the past decades, it has been reported that $\mathrm{ZnS}$ nanomaterials show a good performance in the field of hydrogen evolution from $\mathrm{H}_{2} \mathrm{O},{ }^{6}$ the degradation of organic substrates, ${ }^{7}$ the dehalogenation of halogenated benzene, etc. ${ }^{8,9}$

For nanomaterials, the size, morphology, and phase composition are believed to be major factors determining the activity

\footnotetext{
${ }^{a}$ Key Laboratory for Anisotropy and Texture of Materials (MOE), Northeastern University, Shenyang 110819, P. R. China. E-mail: qingw@smm.neu.edu.cn

${ }^{b}$ Materials and Process Simulation Center, California Institute of Technology, Pasadena, CA 91125, USA

† Electronic supplementary information (ESI) available. See DOI: 10.1039/ c3ср50330e
}

of the photocatalyst. To decrease the distance that photogenerated carriers have to migrate to reaction sites on the surface, tremendous efforts have been made to reduce the sizes and enhance the surface-to-volume ratio of $\mathrm{ZnS}$ nanostructures, such as ZnS based nanoparticles, ${ }^{10}$ 1D nanobelts, ${ }^{11,12}$ nanotube, ${ }^{13,14}$ and complex 3D nano-architectures. ${ }^{15-17}$ These samples were separately fabricated with either the ZB phase, ${ }^{12} \mathrm{WZ}$ phase, ${ }^{11,15}$ or hybrid structures including two phases. ${ }^{16}$

In the case of $\mathrm{TiO}_{2}$, it is clear that anatase $\mathrm{TiO}_{2}$ shows a quite better catalytic activity than rutile $\mathrm{TiO}_{2} \cdot{ }^{18,19}$ To our knowledge, it has been scarcely reported whether different phase structures affect the photocatalytic performance of $\mathrm{ZnS}$ photocatalyst. The type of crystal surface is another influencing factor, recent research shows anatase nanoparticles with a majority of (001) reactive facets. ${ }^{20}$ In addition, strongly anisotropic photocatalytic activity of hematite $\left(\mathrm{Fe}_{2} \mathrm{O}_{3}\right)$ has been observed, which is up to 4-orders of magnitude higher within (0001) basal planes than that orthogonal to them. ${ }^{21}$ Whether such an anisotropy in photocatalytic activity exits in the ZnS system is also under debate. In short, the structure factors and anisotropic activity affecting the photocatalytic efficiency of $\mathrm{ZnS}$ catalyst are still open for investigation. 
In this study, we aim to illustrate the photocatalytic efficiency of ZB and WZ phases, for both in the bulk and at polar surfaces, by means of first-principles calculations based on density functional theory (DFT). The electronic structures of ZB and $\mathrm{WZ}$ phases of bulk ZnS were firstly investigated, and thus the obtained theoretical conductivity ratio of two phases shows a temperature-dependent nature. Furthermore, the minimumenergy paths (MEP) for hydrogen generation from water splitting were presented at ZB and WZ different polar surfaces with Zn or $S$ terminated, respectively. Finally, to simulate the electron excited states activated by photo-irradiation, electronic triplet states were introduced during the MEP calculations. Thus, from points of thermodynamics, electronic transport ability, and chemical kinetics, the difference in photocatalytic efficiency between $\mathrm{ZB}$ and WZ phases is basically understood.

\section{Computational models and methods}

The bulk geometry optimization and electronic properties have been carried out using the ab initio total-energy and molecular dynamics program VASP (Vienna ab initio simulation package). To validate our calculations, different exchange-correlation functionals, e.g., the PW91, ${ }^{22,23} \mathrm{PBE},{ }^{24}$ and B3LYP hybrid functional, ${ }^{25}$ were adopted to perform bandgap and electronic state calculations. An expansion of the electronic wave functions in plane waves with a kinetic-energy cutoff of $600 \mathrm{eV}$ was used. Brillouin-zone integrations were performed using $11 \times 11 \times 11$ Monkhorst-Pack $k$-point meshes ${ }^{26}$ and the Methfessel-Paxton technique $^{27}$ with a $0.05 \mathrm{eV}$ smearing of the electron levels. The relaxation of geometry was carried out to ensure absolute convergence of the total Hellmann-Feynman force with respect to the structural degrees of freedom to a precision of up to $0.1 \mathrm{meV} \AA^{-1}$.

Based on a smoothed Fourier interpolation of the calculated bands, a semi-classic conductivity ratio of $\mathrm{WZ}$ and $\mathrm{ZB}$ phases was evaluated using the BoltzTraP code, ${ }^{28}$ which in principle should be exactly within the frame of the Boltzmann theory. Phonon frequencies in the bulk $\mathrm{ZnS}$ system were calculated based on the small displacement method, as implemented in the PHON code. ${ }^{29}$

This study considers the polar surfaces zinc blende (111) and wurtzite (0001) in ZnS system. For the purpose of describing these faces, the lattices are best considered as a series of Zn-S double layers. Zinc blende (111) differs from wurtzite (0001) only by virtue of the fact that alternate (111) double layers are rotated through $180^{\circ}$ about the [111] axis. For the WZ phase, (0001) is Zn-terminated and (0001) is S-terminated; for the ZB phase, (111) is Zn-terminated and ( $\overline{1} \overline{1} \overline{1})$ is S-terminated. The top and side views of the structures are shown in Fig. 1. The $\mathrm{Zn}$ or S-terminated polar surfaces were represented by a slab model with six Zn-S double layers and a $15 \AA$ vacuum region. Both zinc blende and wurtzite slab supercells contain 48 atoms. To relax the polar surfaces, we fix one bottom $\mathrm{Zn}$-S double layer and free the upper five double layers.

In a complete artificial photosynthesis system, two molecules of water are photochemically split into $\mathrm{H}_{2}$ (reductive side)

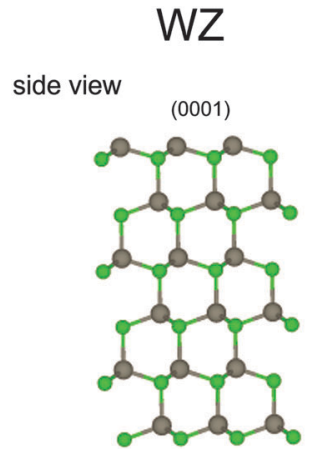

$(000-1)$

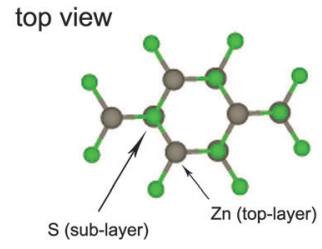

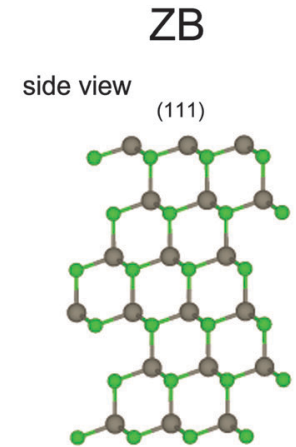

$(-1-1-1)$

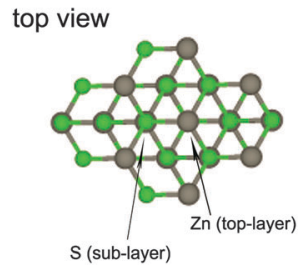

Fig. 1 Wurtzite and zincblende $\mathrm{ZnS}$ lattices. Polar faces are designated. The gray (large) spheres are $\mathrm{Zn}$ atoms; the green (small) spheres are $\mathrm{S}$ atoms.

and $\mathrm{O}_{2}$ (oxidative side). ${ }^{30,31}$ However, when studying the reductive half-reaction it is common for a sacrificial electron donor to be used to optimize catalysts for hydrogen production. ${ }^{32}$ The climbing-image nudged elastic band (cNEB) method, ${ }^{33,34}$ in which the highest energy image is driven up to the saddle point, was used to determine energy barriers of a possible chemical reaction path for water splitting and hydrogen generation. The Brillouin zone was sampled with $5 \times 5 \times 1$ $k$-point mesh, and a force-based conjugated gradient method with an accuracy up to $0.005 \mathrm{eV} \AA^{-1}$ for every image was used to optimize the geometry. In addition to the ground states, electronic triplet states calculations were performed by turning one pair of spin-opposite electrons to two independent spinparallel electrons.

\section{Results and discussion}

\section{Electronic structures of intrinsic bulk phases}

From a theoretical point of view, studying the electronic structures of the two phases of bulk ZnS constitutes no doubt a first necessary step. The structural stabilities of relaxed bulk $\mathrm{ZB}$ and $\mathrm{WZ}$ phases were confirmed via thermodynamic and lattice dynamics investigations. We calculated the formation energy with respect to the metal zinc and sulfur (orthorhombic $\alpha$-S8) based on the reaction $\mathrm{Zn}+\mathrm{S}=\mathrm{ZnS}$. The resulting negative formation energies of $\mathrm{ZB}$ and $\mathrm{WZ}$ are -1.76 and $-1.74 \mathrm{eV} /$ atom, respectively, indicating that both phases are thermodynamically stable. Dynamic stabilities of two phases are evaluated by the phonon dispersion relations, as shown in Fig. 2. Both ZB and WZ phases are stable as there are no imaginary modes. The lower frequencies of the total density of states (DOSs) should be dominated by lattice dynamics of heavy $\mathrm{Zn}$ atoms and higher frequencies by light $\mathrm{S}$ atoms. Moreover, there are theoretical gaps, 
(a)

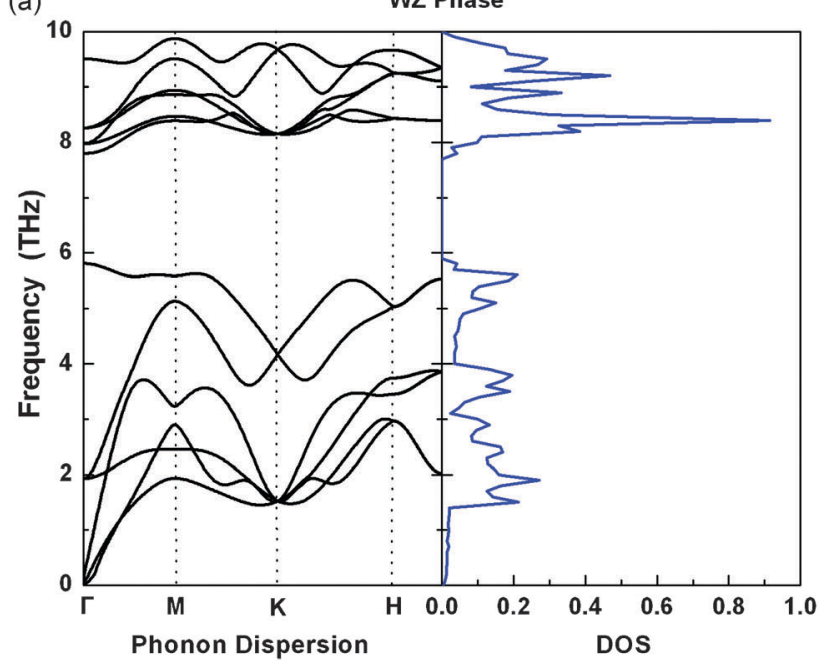

(b)

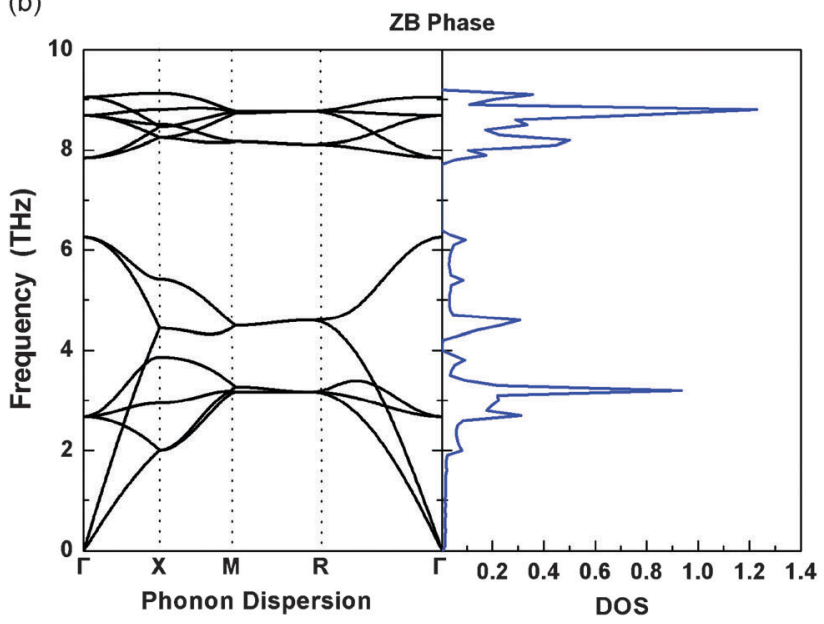

Fig. 2 The phonon dispersion relations and DOSs of WZ (a) and ZB (b) phases.

6-7.6 $\mathrm{THz}$ in $\mathrm{WZ}$ phase and 6.5-7.6 $\mathrm{THz}$ in $\mathrm{ZB}$ phase, in phonon frequencies.

Various studies on DFT calculations for ZnS yield band gaps that are either overestimated ${ }^{35}$ or, more commonly, underestimated. ${ }^{36-38}$ It has been reported that $\mathrm{GW}^{39}$ approximations can produce a band gap of experimental quality, but the cost of the calculation is expensive. In this paper we adopt PW91, PBE, and B3LYP exchange-correlation functionals, and focus mainly on the self-consistency between these methods. The band gaps and projected density of states (PDOSs) of the intrinsic bulk WZ and $\mathrm{ZB}$ phases are illustrated in Fig. 3. Together with the equilibrium lattice parameters by PW91, PBE, and B3LYP, conduction band minimum (CBM) and valence band maximum (VBM) at the $\Gamma$ point are listed in Table 1 . To compare energy levels obtained by different functions, we introduce the average electrostatic potential and the core level shift between ZB and WZ models are erased.

It is clear that both $\mathrm{ZB}$ and $\mathrm{WZ}$ have a direct band gap at the $\Gamma$ point. The PW91 band gaps of $\mathrm{ZB}$ and $\mathrm{WZ}$ are 2.02 and $2.08 \mathrm{eV}$, PBE gaps are 2.03 and $2.09 \mathrm{eV}$, and B3LYP gaps are
3.05 and 3.11, respectively. Compared with experimental gaps, i.e. $3.72^{3} \mathrm{eV}$ for $\mathrm{ZB}$ and $3.77^{4} \mathrm{eV}$ for $\mathrm{WZ}$, current calculations underestimate the band gaps in the experimental ZnS system. However, the theoretical difference in the band gaps $(0.06 \mathrm{eV})$ between WZ and ZB agrees well with the experimental difference $(0.05 \mathrm{eV})$.

Information on CBM and VBM is very important for discussing the photochemical reaction mechanism in the photocatalyst. We can regard CBM as the electron affinity. The higher the level of CBM, the stronger the ability of losing electrons or reducing ability. On the other hand, since the hole is created at VBM and attracts the electrons from the material that is in contact with or adsorbed to the system, the ability of attracting electrons or oxidative activity would increase if the VBM were located at a lower level. In the current calculations, PW91, PBE, and B3LYP CBMs in the WZ phase are higher than that in $\mathrm{ZB}$ phase by $0.06,0.06$, and $0.08 \mathrm{eV}$, respectively, while VBMs are almost the same with that in $\mathrm{ZB}$ phase. Considering the differences in the location of CBM and VBM levels between $\mathrm{ZB}$ and $\mathrm{WZ}$ phase, the $\mathrm{WZ}$ phase has a slightly stronger reducing power than the $\mathrm{ZB}$ phase.

The PDOSs show similar electronic features for ZB and WZ phases around the Fermi level $E_{\mathrm{F}}$. The upper valence band, lying just below the $E_{\mathrm{F}}$, is essentially an $\mathrm{S}-3 p$ orbital with clear hybrids with $\mathrm{Zn}-3 p, 3 d$ states. A conduction band of $\mathrm{Zn}-4 s$ character, with contribution from the S-3p level is observed above $E_{\mathrm{F}}$. Thus, a crude model for the generation of electronhole pairs in the ZnS system can be regarded as the break of the $\mathrm{S}_{3 p}-\mathrm{Zn}_{3 p}$ bonds and formation of the $\mathrm{S}_{3 p}-\mathrm{Zn}_{4 s}$ bonds.

Even if the photogenerated electrons and holes possess thermodynamically sufficient potentials for the photocatalytic reaction, low charge mobility will lead to a high electron-hole recombination rate before carriers are transported to the active sites on the surface. Thus the conductivity essentially affects the photocatalytic efficiency of the photocatalytic materials. Fig. 4 shows the conductivity ratio of intrinsic bulk WZ and ZB structures. The curves indicate that the conductivity ratio is temperature-dependent. At low temperature, i.e. below $200 \mathrm{~K}$ for PW91 and PBE and below $300 \mathrm{~K}$ for B3LYP, the conductivity of $\mathrm{WZ}$ phase is superior to that of the $\mathrm{ZB}$ phase. However, the conductivity of the $\mathrm{ZB}$ phase will surpass that of the $\mathrm{WZ}$ phase above $300 \mathrm{~K}$. As the temperature increases, the asymptotic conductivity ratio is close to the value of the golden ratio 0.62 .

Recently, Hong et al. synthesized ZB and WZ nano-structures at temperatures above $150{ }^{\circ} \mathrm{C} .{ }^{42}$ They found that the photocatalytic activity of the $\mathrm{ZB}$ phase is stronger than that of $\mathrm{WZ}$ phase. However, the reasons for this phenomenon are still unclear. By the present calculations, we propose that it would be related to the better performance in conductivity of $\mathrm{ZB}$ phase at high temperature.

\section{Hydrogen generation due to water splitting on polar surfaces}

Since the surface chemistry and structure of the photocatalysts are very important to determine the catalytic activity, the $\mathrm{ZB}$ (111)-Zn and (1̄1̄1)-S-terminated surfaces, as well as WZ (0001)Zn and (0001)-S-terminated surfaces are seriously considered in 

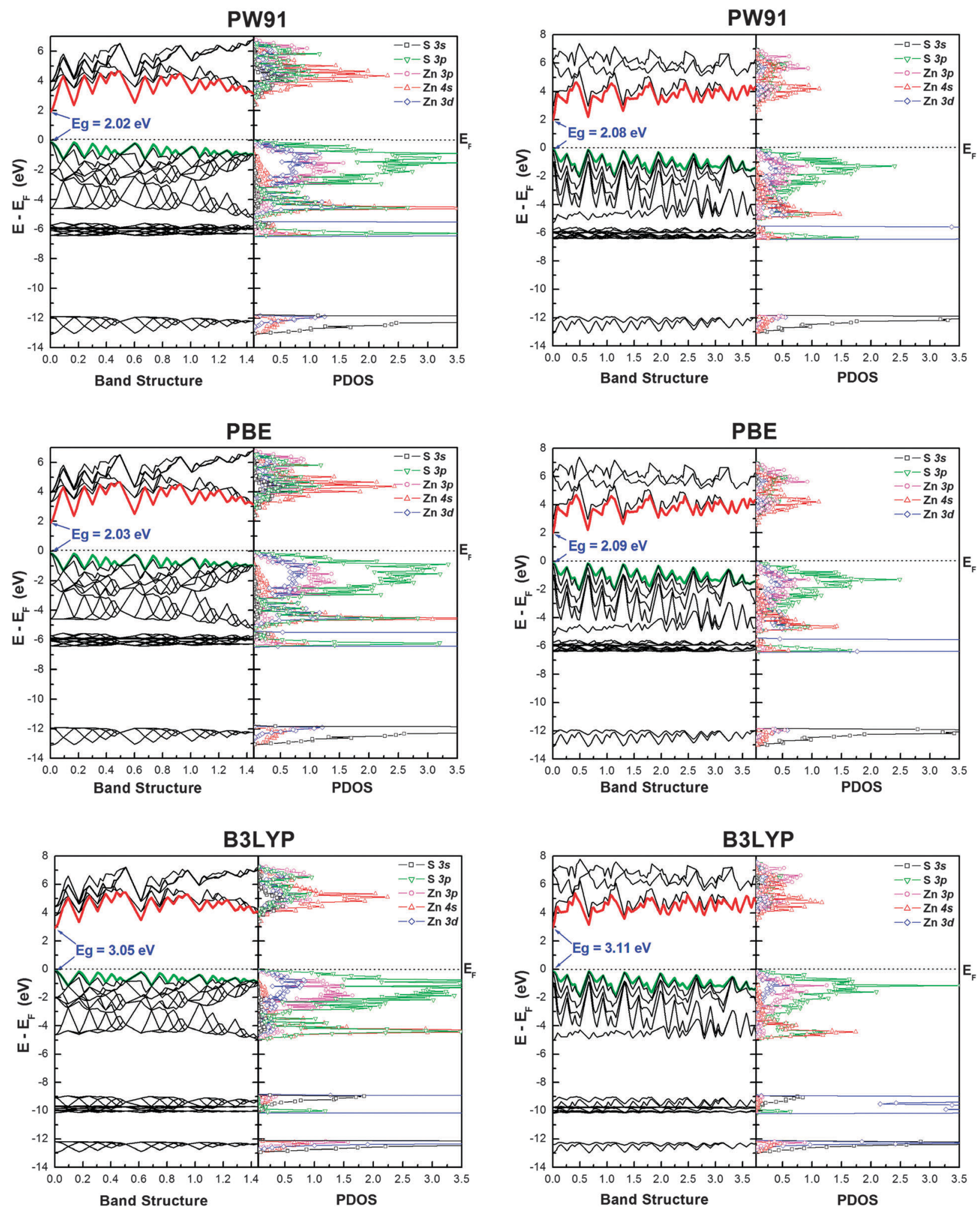

Fig. 3 PW91, PBE, and B3LYP calculated band structures and PDOSs of the ZB (left) phase and the WZ (right) phase.

this work. We investigated the thermodynamic stabilities of these polar surfaces via the calculations on the surface energy which is defined as $1 / 2 A\left(E_{\text {total }}-E_{\text {bulk }}\right)$, where $E_{\text {total }}$ is the total energy of the slab, $E_{\text {bulk }}$ is the total energy of the bulk ZnS composed of the same number of atoms, and $A$ is the surface area of the simulation cell. The order of surface energy for polar surfaces is $E$ ZBZn $=1.35 \mathrm{~J} \mathrm{~m}^{-2}<E$ WZZn $=1.37 \mathrm{~J} \mathrm{~m}^{-2}$ $<E \mathrm{WZS}=1.38 \mathrm{~J} \mathrm{~m}^{-2}<E \mathrm{ZBS}=1.39 \mathrm{~J} \mathrm{~m}^{-2}$, where superscripts 
Table 1 The DFT calculated and experimental lattice constants (in $\AA$ ) of ZB and WZ bulk phases, together with the conduction band minimum (CBM), valence band maximum (VBM) and Fermi level $E_{\mathrm{F}}$ (in eV) at $\Gamma$ point by PW91, PBE and B3LYP calculations

\begin{tabular}{|c|c|c|c|c|c|c|c|c|c|c|c|}
\hline Method & \multicolumn{5}{|l|}{ ZB phase } & \multicolumn{6}{|c|}{ WZ phase } \\
\hline B3LYP & 5.33 & 151.56 & 0.58 & 3.64 & 0.70 & 3.77 & 6.17 & 75.91 & 0.59 & 3.70 & 0.73 \\
\hline PBE & 5.44 & 161.37 & 1.90 & 3.93 & 2.03 & 3.85 & 6.30 & 80.77 & 1.91 & 4.01 & 2.04 \\
\hline Exp. & $5.41^{a}$ & $158.25^{a}$ & - & - & - & $3.82^{b}$ & $6.26^{b}$ & $79.23^{b}$ & - & - & - \\
\hline
\end{tabular}

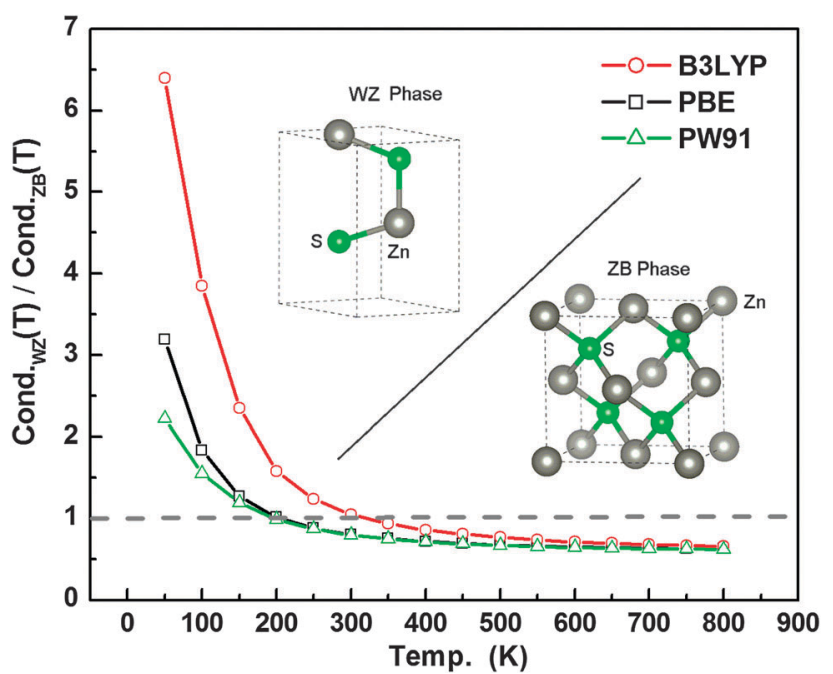

Fig. 4 PW91, PBE, and B3LYP calculated conductivity ratio between WZ and ZB phases.

denote phase and subscripts denote the atom with which the polar surface is terminated. Thus, Zn-terminated polar surfaces are generally more stable than S-terminated polar surfaces.

To explore the chemical activities of different polar surfaces, we studied one possible reaction pathway of hydrogen generation from water splitting on these surfaces using the PBE exchange-correlation functional through DFT calculations on the MEP.

Firstly, we optimized the $\mathrm{H}_{2} \mathrm{O}$ molecule at various high symmetry points on the $\mathrm{ZnS}$ polar surfaces (i.e., $\mathrm{H}_{2} \mathrm{O}$ at bridge sites, on-top sites, and hollow sits). On the Zn-terminated surfaces, we found that $\mathrm{H}_{2} \mathrm{O}$ at the top site of $\mathrm{Zn}$ on the ZB-(111) and WZ-(0001) surfaces is the most stable adsorption configuration, which is shown in Fig. 5 (IS). The $\mathrm{O}$ atom is 2.142 and $2.149 \AA$ from the on-top $\mathrm{Zn}$ atoms on ZB-(111) and WZ-(0001) surfaces, respectively, with two $\mathrm{H}-\mathrm{O}$ bonds pointing toward the vacuum. The adsorption energy has been calculated using the formula $E_{\text {ads }}=E_{\text {tot }}-\left(E_{\text {sur }}+E_{\mathrm{H}_{2} \mathrm{O}}\right)$, where $E_{\text {tot }}$ is the total energy of the combined system $\left(\mathrm{H}_{2} \mathrm{O}\right.$ bound to surfaces), $E_{\text {sur }}$ is the energy of the surface alone, and $E_{\mathrm{H}_{2} \mathrm{O}}$ is the energy of a solo $\mathrm{H}_{2} \mathrm{O}$ molecule. $E_{\text {ads }}$ of $\mathrm{H}_{2} \mathrm{O}$ on the $\mathrm{Zn}$-terminated faces are -1.06 and $-0.98 \mathrm{eV}$ for ZB-(111) and WZ-(0001), respectively. On the S-terminated surfaces, the global minimum is $\mathrm{H}_{2} \mathrm{O}$

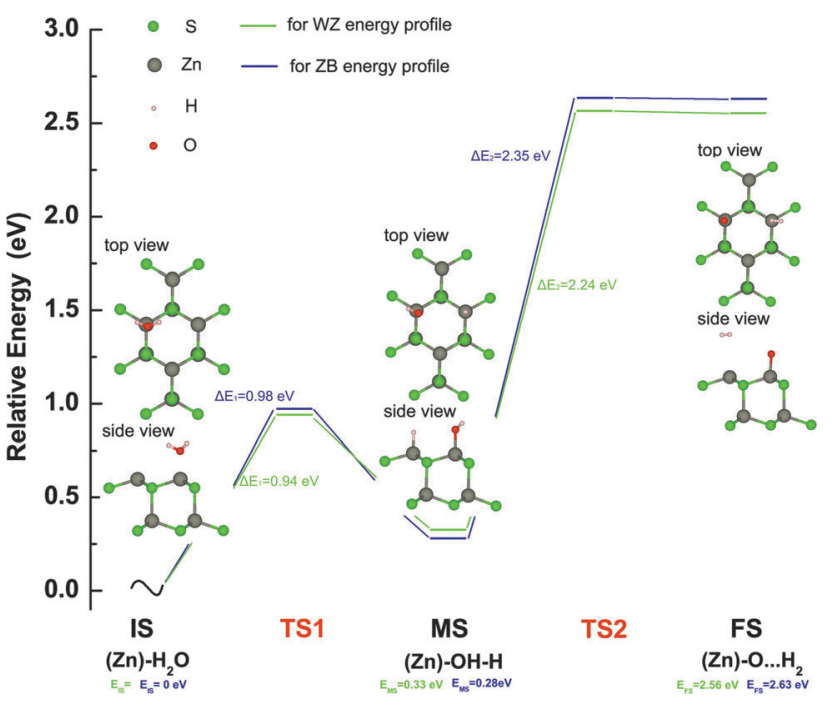

Fig. 5 NEB calculated reaction path and corresponding energetics for the hydrogen generation from water splitting on the Zn-terminated polar surfaces. The energy differences are referred to the initial state (IS). The rate-determining step on the Zn-surfaces has a barrier of 2.35 and $2.24 \mathrm{eV}$ for ZB (111) and WZ (0001), respectively.

above the hollow site surrounded by three $\mathrm{S}$ atoms, which is shown in Fig. 6 (IS). The $\mathrm{H}_{2} \mathrm{O}$ stands with two O-H bonds pointing toward two surface $\mathrm{S}$ atoms at distances of 2.905 and $2.928 \AA$ for $\mathrm{ZB}-(\overline{1} \overline{1} \overline{1}), 3.175$ and $3.166 \AA$ for WZ-(0001) $)$. The adsorption energy $E_{\text {ads }}$ of $\mathrm{H}_{2} \mathrm{O}$ on the S-terminated polar surfaces is -0.21 and $-0.23 \mathrm{eV}$ for ZB-( $(\overline{1} \overline{1} \overline{1})$ and WZ-(0001), respectively. Generally speaking, the structures of $\mathrm{H}_{2} \mathrm{O}$ on the $\mathrm{Zn}$-terminated faces have lower adsorption energies than on the S-terminated surfaces, indicating that $\mathrm{Zn}$-terminated faces have more electronic interaction with water molecule.

Then, a NEB is constructed with the initial state (IS) consisting of polar surfaces with water adsorbed in the preferred sites. The final state (FS) end with a hydrogen molecule above the polar surfaces beyond the interaction distance. Intermediate images along the NEB were linearly interpolated between the initial and final states.

The MEP of the reaction pathway for the Zn-terminated surfaces is shown in Fig. 5. The energy barrier of the first $\mathrm{H}$ atom splitting from water is 0.98 and $0.94 \mathrm{eV}$ for ZB (111) and WZ (0001), respectively. The second $\mathrm{H}$ splitting from $\mathrm{OH}$ needs 2.35 and $2.24 \mathrm{eV}$ for ZB (111) and WZ (0001), respectively. 
$\mathrm{H}_{2}$ can then form directly after the second rate-determining barrier is surmounted. The distance between the $\mathrm{H}_{2}$ molecule and the Zn-Surfaces increases to 4.048 and $3.941 \AA$ for ZB (111) and WZ (0001), respectively, at which point $\mathrm{H}_{2}$ can easily desorb from the $\mathrm{Zn}$-terminated surfaces. Globally, $\mathrm{H}_{2}$ production on $\mathrm{Zn}$ surfaces is an endothermic reaction with heats of reaction of 2.63 and $2.56 \mathrm{eV}$ for ZB (111) and WZ (0001), respectively.

The MEP of the reaction pathway for the S-terminated surfaces is shown in Fig. 6. The first $\mathrm{H}$ atom splitting yields an $\mathrm{OH}$ radical and simultaneously forms a $\mathrm{S} @-\mathrm{OH}$ bond. The first energy barriers are 1.45 and $1.53 \mathrm{eV}$ for $\mathrm{ZB}(\overline{1} \overline{1} \overline{1})$ and WZ (0001), respectively, which is much larger than that on the Zn-terminated surfaces. The reaction step involving the second $\mathrm{H}$ splitting from $\mathrm{OH}$ has a high barrier of $2.97 \mathrm{eV}$, for both ZB ( $\overline{1} \overline{1} \overline{1})$ and WZ (0001). The $\mathrm{H}$ atoms then form two S-H bonds on the S-surface which lead to an intermediate state MS2. The following $\mathrm{S} @$.... $\mathrm{H}_{2}$ dissociations have very low barriers of 0.06 and $0.05 \mathrm{eV}$ for $\mathrm{ZB}(\overline{1} \overline{1} \overline{1})$ and $\mathrm{WZ}(000 \overline{1})$, respectively. The distances between the $\mathrm{H}_{2}$ molecule and the S-Surfaces are 3.862 and $3.799 \AA$ for $\mathrm{ZB}(\overline{1} \overline{1} \overline{1})$ and $\mathrm{WZ}(000 \overline{1})$, respectively. Compared with $\mathrm{Zn}$-surfaces, there is an obvious decrease in total energy after $\mathrm{H}_{2}$ desorbs from the S-surfaces, which results in a lower heat of reaction of 2.15 and $2.18 \mathrm{eV}$ for $\mathrm{ZB}(\overline{1} \overline{1} \overline{1})$ and

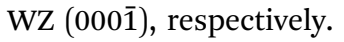

The MEP analysis shows that the rate-determining barriers of water splitting on $\mathrm{Zn}$-terminated polar surfaces is lower than that on corresponding S-terminated polar surfaces, suggesting that the Zn-terminated polar surface is chemically active, while the S-terminated polar surface is relatively inert in the reaction of photolysis of water into hydrogen.

Finally, to explore the reaction mechanism for water splitting on $\mathrm{ZnS}$ polar surfaces, charge transfer between $\mathrm{H}_{2} \mathrm{O}$ and surfaces was studied on the basis of DOSs and bonding charge

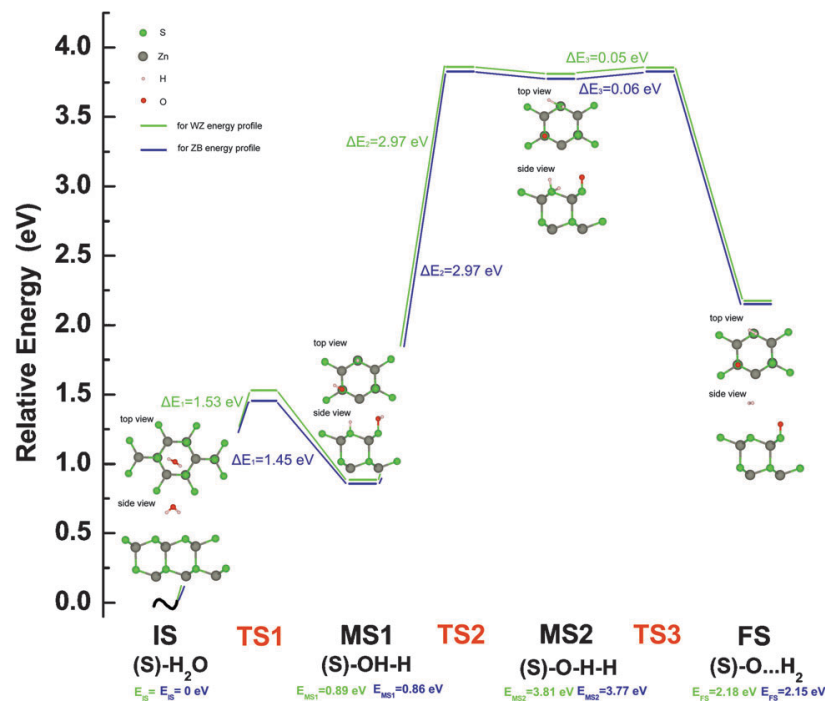

Fig. 6 NEB calculated reaction path and corresponding energetics for the hydrogen generation from water splitting on the S-terminated polar surfaces. The energy differences are referred to the initial state (IS). The ratedetermining step on the S-surfaces has a barrier of $2.97 \mathrm{eV}$ for both ZB ( $\overline{1} \overline{1} \overline{1})$ and WZ (0001).
$\Delta \rho$ density calculations. The bonding charge density was evaluated with the expression: $\Delta \rho(r)=\rho_{\mathrm{X} @ \text { sur }}(r)-\rho_{\text {sur }}(r)-\rho_{\mathrm{X}}(r)$, where $\rho_{\mathrm{X} @ \text { sur }}(r), \rho_{\text {sur }}(r)$, and $\rho_{\mathrm{X}}(r)$ are the charge density of the whole system, the isolated surface, and the adsorbate, respectively. In the text, we only present the DOSs and $\Delta \rho$ of states along the reaction pathway on the $\mathrm{WZ}$ polar surfaces, those on the ZB polar surfaces are similar to that on the WZ surfaces and can be found in the ESI. $†$

The DOSs and bonding charge analysis of states on the WZ (0001) Zn-terminated surface (Fig. 7) reveals that the O atom has an electron interaction with $\mathrm{Zn}$ in IS, however, the bonding electrons are localized in the deep shell and hardly contribute to the electron transfer. In MS, it is clear that the significant electronic coupling between adsorbate and surface appears at the Fermi energy $E_{\mathrm{F}}$. The whole electronic structures exhibit a $p$-type because the $E_{\mathrm{F}}$ locates at the VBM of energy bands, hinting that bonding electrons have not been activated. Thus, photo-assistance should be needed just after MS for the electronic excitation and subsequent conduction electron transfer between adsorbate and surface. Electron coupling occurs only between the $\mathrm{O}$ atom and the surface in FS, while $\mathrm{H}$ has no action with the $\mathrm{O}$ atom and has desorbed from surface.

The DOSs and bonding charge of the WZ (0001) S-terminated surface (Fig. 8) show a reaction mechanism totally different to that of $\mathrm{Zn}$ surface. A remarkable feature of weak electronic interaction between the surface and adsorbate was first found in IS, in which DOSs of $\mathrm{H}$ and $\mathrm{O}$ atoms are almost the same as that in a solo water molecule, indicating a nonbonding property between water and the surface. More importantly, the DOSs in MS1 show $n$-type because of the $E_{\mathrm{F}}$ at the CBM of energy bands, which means that the bonding electrons around $E_{\mathrm{F}}$ have been activated and delocalized and conductive activity. This state can only be achieved through photoradiation on the S-terminated surface before MS1. In MS2, the electronic banding is mainly found between $\mathrm{H}$ atoms and surface, which facilitate the reducing of $\mathrm{H}_{2}$ molecule. Finally, $\mathrm{H}_{2}$ desorbs from the S-polar surface in FS.

The MS on the Zn-surface, as well as MS1 on the S-surface, are the expressions for the first $\mathrm{H}$ atom splitting from water. The above analysis reveals the fact that photo-radiation is not need for the first $\mathrm{H}$ splitting on the $\mathrm{Zn}$-terminated surface, while necessary for those on the S-terminated surface. This can also be understood because of the high-energy barrier $(1.53 \mathrm{eV})$ for the first $\mathrm{H}$ atom splitting from water on the $\mathrm{S}$-surface. The chemical kinetics studies suggest that Zn-terminated polar surfaces be more chemically active than S-terminated polar surfaces.

It should be noticed that the discussion above is based on the DFT in the electron ground state. We propose that WZ (0001) possessing slightly a better performance in photocatalytic activity than ZB (111) may relate to its higher conductivity at low temperature, as discussed in Section 3.1. The conductivity values of two phases will reverse with the increase of temperature and chemical activity of ZB (111) may surpass that of WZ (0001). 
WZ-Zn Surf. -IS

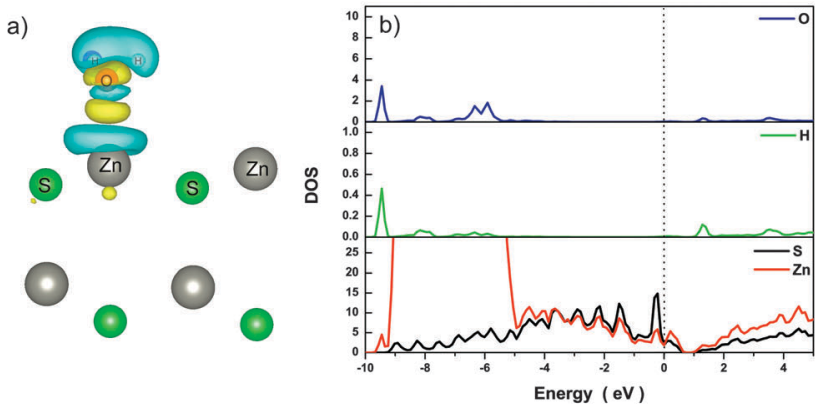

WZ-Zn Surf.-MS

c)
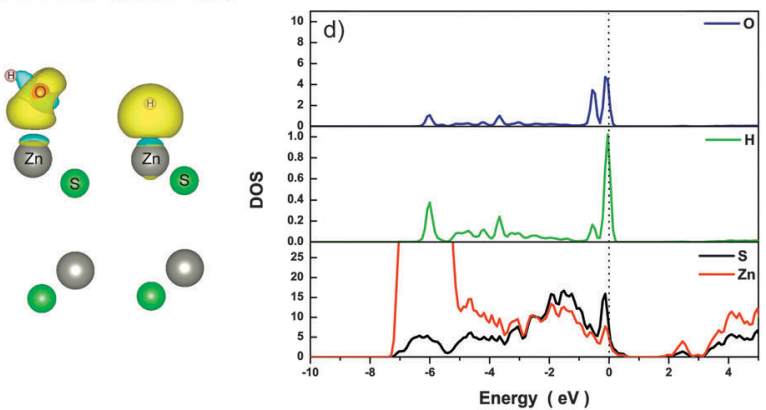

WZ-Zn Surf.-FS e)

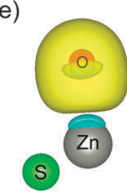

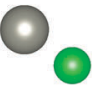

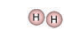

$\mathrm{Zn}$

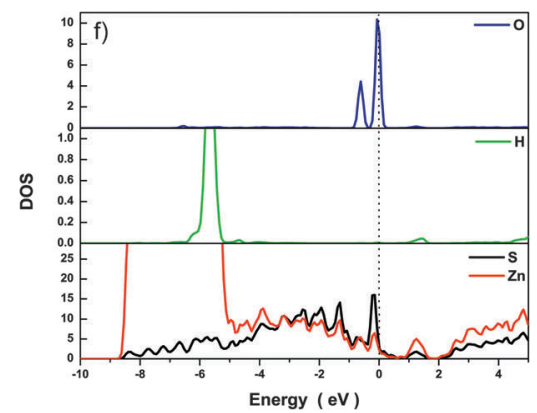

Fig. 7 Calculated DOSs and bonding charge density of states along the MEP path on the WZ (0001) surface. a) and b) for IS, c) and d) for MS, and (e) and (f) for FS. Energies are referred to the Fermi level, which is marked by a vertical dash line.

It is significant to consider factors that may affect the reaction process with high reaction barriers for water splitting on $\mathrm{ZnS}$ polar surfaces. We speculated that it might be related to the defects on polar surfaces and photo-radiation.

We first studied point vacancy defects on all concerned polar surfaces by removing an outer layer atom from its lattice site. The defect formation energy $E_{\text {form }}$ was evaluated with the formula as $E_{\text {form }}=E_{\text {def }}+E_{\text {atom }}-E_{\text {sur }}$, where $E_{\text {def }}$ is the total energy of the fully relaxed slab containing a vacancy, $E_{\text {atom }}$ is the reference energy for the defect atom, and $E_{\text {sur }}$ is the energy of an ideal perfect surface. The calculated defect formation energies $E_{\text {form }}$ of Zn-terminated faces are -0.65 and $-0.64 \mathrm{eV}$ for ZB-(111) and WZ-(0001), respectively, and that of S-terminated faces are 1.54 and $1.56 \mathrm{eV}$ for $\mathrm{ZB}-(\overline{\mathbf{1}} \overline{1} \overline{1})$ and WZ-(0001), respectively. It is surprising that Zn-defect polar surfaces are more stable than Zn-perfect polar surfaces, which also indicates that Zn-perfect polar surfaces are more
WZ-S Sur.-IS a)

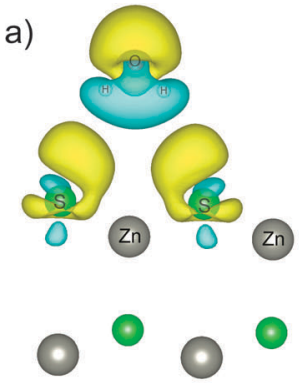

WZ-S Sur.-MS1

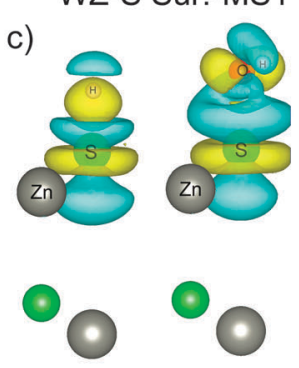

WZ-S Sur.-MS2

e)
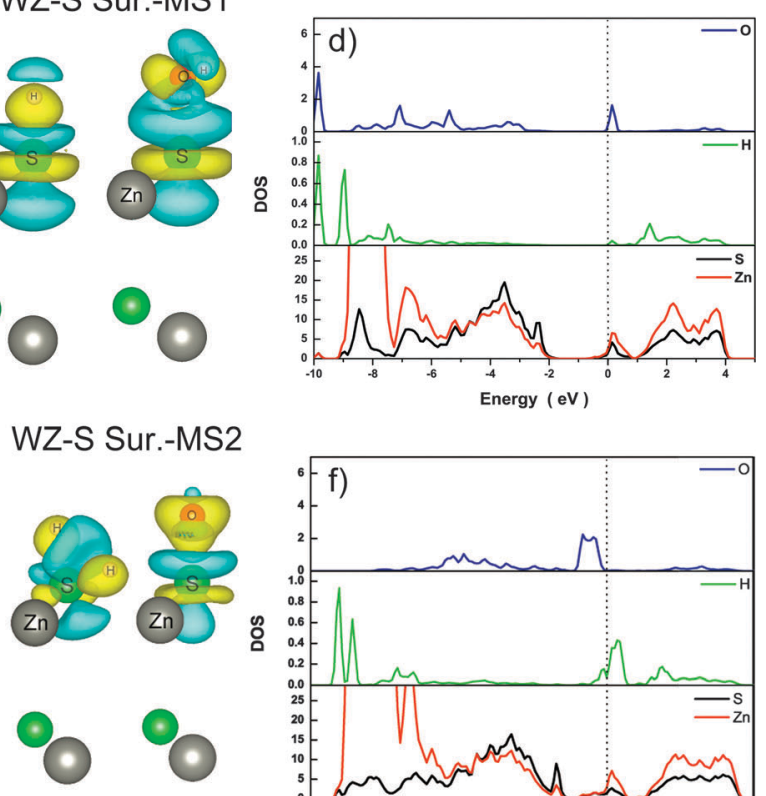

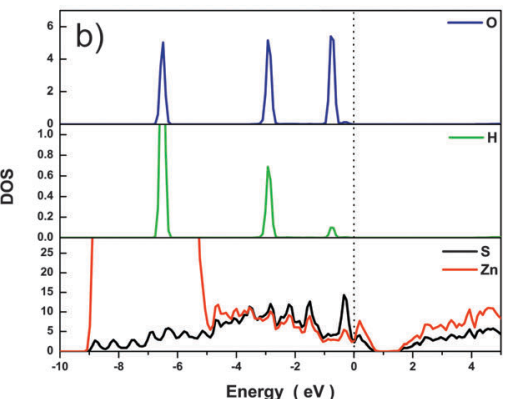

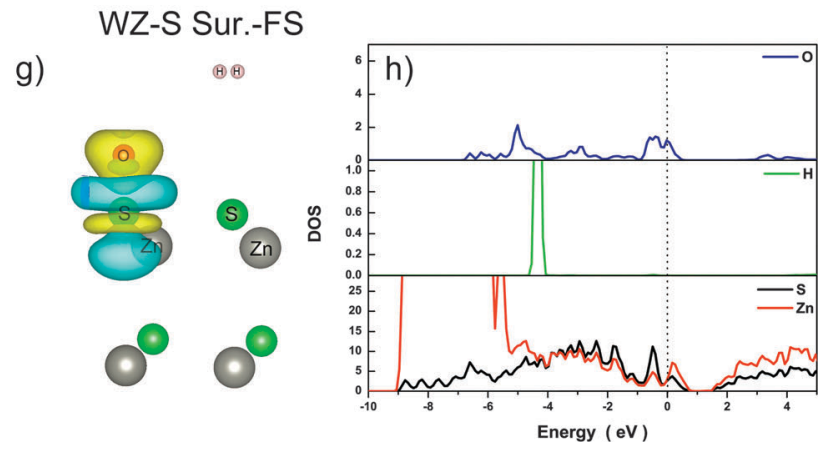

Fig. 8 Calculated DOSs and bonding charge density of states along the MEP path on the WZ (0001) surface. a) and b) for IS, c) and d) for MS1, (e) and (f) for MS2, and g) and h) for FS. Energies are referred to the Fermi level, which is marked by a vertical dash line.

chemically active than Zn-defect polar surfaces. However, S-defect polar surfaces can hardly form due to the high defect formation energy.

Combining with previous DFT calculations, it is found that $\mathrm{H}_{2} \mathrm{O}$ at the top site of $\mathrm{Zn}$ on the ZB-(111) or WZ-(0001) surfaces is the most stable adsorption configuration. So if a $\mathrm{Zn}$ atom is lost, the binding between $\mathrm{H}_{2} \mathrm{O}$ and surfaces would be looser and transport of electrons more difficult. In addition, the 
Zn-defect states tend to capture photons and weaken the production of electron-hole pairs. Therefore, we propose that the Zn-defect on the polar surface is unfavorable to the photocatalytic reaction of water splitting due to its low chemical activity, weak bonding to the water molecule, and trapping photons.

In a recent paper, ${ }^{42}$ it was first reported that the enhancement of the crystallinity of the $\mathrm{ZnS}$ material can lead to an increase of the photocatalytic efficiency. The conclusion experimentally proves that ideal perfect surfaces are more conducive to the photocatalytic reaction than defect surfaces for $\mathrm{ZnS}$ material. To fully understand the effect of defects on the photocatalytic activity of $\mathrm{ZnS}$, more computational designs should be needed in our next step works.

Photo-radiation induced photochemical reactions involving electronic excitation should be discussed on the basis of real-time, real-space excitation calculations, such as timedependent density functional theory (TD-DFT) or the GW/BSE method. ${ }^{43}$ It is still difficult to fulfill calculations of the photoinduced reaction pathway in such extended systems using these methods. However, it does not prevent us from using the approximation method to take a glance at the excited photocatalytic reaction path. We introduced the electronic triplet state during the calculations on the MEP of hydrogen generation from water splitting on the $\mathrm{Zn}$ - or S-terminated polar surfaces. The results are illustrated in Fig. 9 and 10, respectively.

It is clear that in MEPs on the Zn-terminated surfaces, the rate-determining barriers reduced significantly from 2.35 and $2.24 \mathrm{eV}$ to 2.11 and $2.06 \mathrm{eV}$ for the $\mathrm{ZB}$ (111) and $\mathrm{WZ}$ (0001), respectively. However, on the S-terminated surfaces, the ratedetermining barriers almost remain unchanged for both $\mathrm{ZB}$ $(\overline{1} \overline{1} \overline{1})$ and $\mathrm{WZ}(000 \overline{1})$. The current calculations support that the Zn-terminated surfaces are photosensitive, while S-terminated surfaces are photoinert. The spin DOSs which presents the population of unpaired electrons can be found in the ESI. $†$

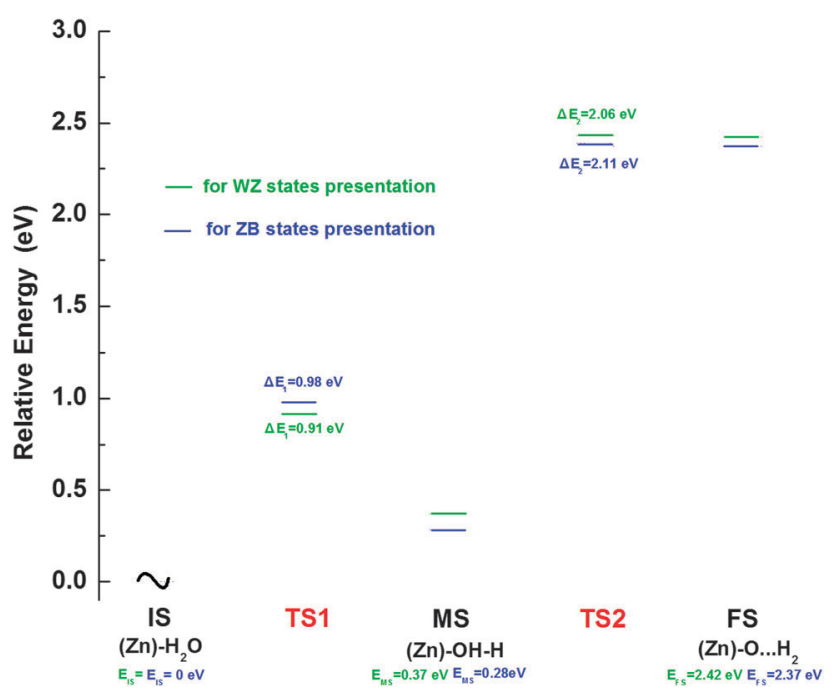

Fig. 9 Electronic triplet energetics for the hydrogen generation from water splitting on the Zn-terminated polar surfaces. The energy differences are referred to the initial state (IS). The rate-determining step on the $\mathrm{Zn}$-surfaces has a barrier of 2.11 and $2.06 \mathrm{eV}$ for ZB (111) and WZ (0001), respectively.

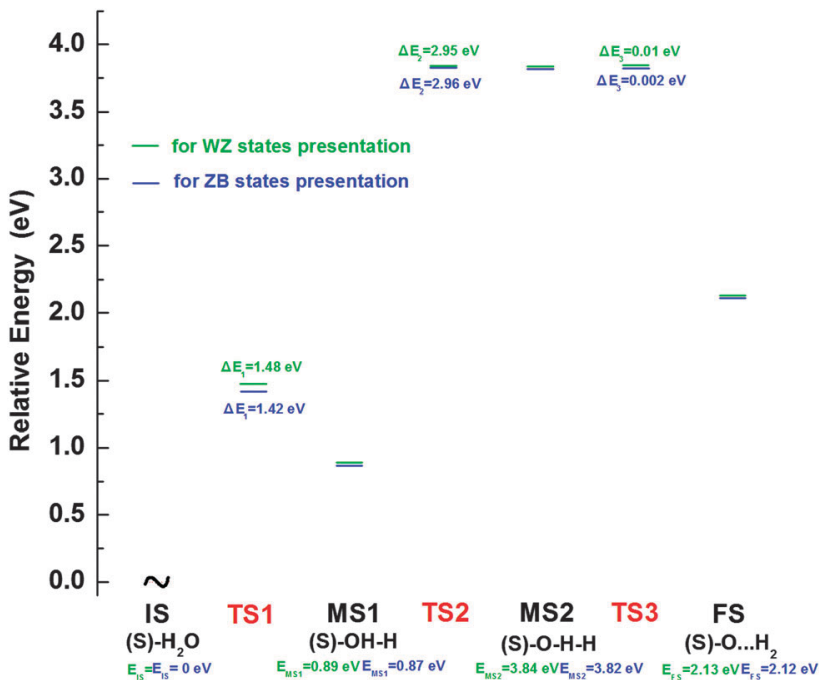

Fig. 10 Electronic triplet energetics for the hydrogen generation from water splitting on the S-terminated polar surfaces. The energy differences are referred to the initial state (IS). The rate-determining step on the S-surfaces has a barrier of 2.96 and $2.95 \mathrm{eV}$ for ZB (111) and WZ (0001), respectively.

\section{Conclusions}

Based on the first-principle calculations, the factors of structures and surface polarity that influence the photocatalytic activity of ZnS family were investigated in this paper. The chemical thermodynamics calculations prove that the WZ phase has a higher reducing ability than the $\mathrm{ZB}$ phase. The investigation of carrier transport ability shows that the conductivities of $\mathrm{WZ}$ and $\mathrm{ZB}$ phases are temperature-dependent. At or above room temperature, the conductivity of the bulk ZB phase surpasses that of the $\mathrm{WZ}$ phase. As the temperature increases, the asymptotic conductivity ratio of $\mathrm{WZ} / \mathrm{ZB}$ is close to the golden ratio 0.62. Furthermore, the fundamental mechanism of hydrogen generation from water splitting is established on the Zn- or S-terminated polar surfaces, respectively. On Zn-faces, two transition states (TSs) with barrier energies of $0.98(0.94)$ and $2.35(2.24) \mathrm{eV}$ have been identified for the ZB (WZ) phase. On S-faces, three transition states have been found with barrier energies of 1.45 (1.53) eV (the first TS), 2.97 (2.97) eV (the second TS), and $0.06(0.05) \mathrm{eV}$ (the third TS) for ZB (WZ) phase. The rate-determining barriers indicate that Zn-terminated polar surfaces are more chemical active than $\mathrm{S}$-terminated polar surfaces in the reaction of hydrogen generation from water splitting. To realize the final hydrogen production, photoassistance is needed for the second $\mathrm{H}$ atom splitting on Zn-surfaces, while necessary for the first $\mathrm{H}$ splitting on S-surfaces. The photo-excited MEP was simulated by means of electronic triplet calculations, showing that Zn-terminated surfaces are more photosensitive than S-terminated surfaces. Our results supports the proposal that structures and surface polarity control plays an important role in improving photocatalytic efficiency of nanostructures in the $\mathrm{ZnS}$ family, thus paving the way to effective molecular engineering of further enhanced ZnS semiconductors for photocatalytic applications. 


\section{Acknowledgements}

The authors gratefully acknowledge financial support by the National Natural Science Foundation of China (no. 51001025 and 51002026), the Fundamental Research Funds for the Central Universities (no. 110405003, 110810001 and 100702001) and National 863 project (2012AA030314).

\section{Notes and references}

1 A. Fujishima and K. Honda, Nature, 1972, 238, 37.

2 M. Grätzel, Nature, 2001, 414, 338.

3 T. K. Tran, W. Park, W. Tong, M. M. Kyi, B. K. Wagner and C. J. Summers, J. Appl. Phys., 1997, 81, 2803.

4 H. C. Ong and R. P. H. Chang, Appl. Phys. Lett., 2001, 79, 3612. 5 A. Kudo and Y. Miseki, Chem. Soc. Rev., 2009, 38, 253.

6 I. Tsuji, H. Kato, H. Kobayashi and A. Kudo, J. Am. Chem. Soc., 2004, 126, 13406.

7 S. Yanagida, H. Kizumoto, Y. Ishimaru, C. Pac and H. Sakurai, Chem. Lett., 1985, 141.

8 S. Yanagida, H. kawakami, Y. Midori, H. Kizumoto, C. Pac and Y. Wada, Bull. Chem. Soc. Jpn., 1995, 68, 1811.

9 S. Kohtani, Y. Ohama, Y. Ohno, I. Tsuji, A. Kudo and R. Nakagaki, Chem. Lett., 2005, 34, 1056.

10 X. Li, C. Hu, H. Liu, J. Xu, B. Wan and X. Wang, Phys. E., 2011, 43, 1071.

11 X. Fang, Y. Bando, M. Liao, T. Zhai, U. K. Gautam, L. Li, Y. Koide and D. Golberg, Adv. Funct. Mater., 2010, 20, 500.

12 Y. Ding, X. D. Wang and Z. L. Wang, Chem. Phys. Lett., 2004, 398, 32.

13 X. Wang, P. Gao, J. Li, C. J. Summers and Z. L. Wang, Adv. Mater., 2002, 14, 1732.

14 H. Zhang, S. Zhang, S. Pan, G. Li and J. Hou, Nanotechnology, 2004, 15, 945.

15 S. Xiong, B. Xi, C. Wang, D. Xu, X. Feng, Z. Zhu and Y. Qian, Adv. Funct. Mater., 2007, 17, 2728.

16 J. Liu, Z. Guo, W. Wang, Q. Huang, K. Zhu and X. Chen, Nanoscale, 2011, 3, 1470.

17 Y. G. Yu, G. Chen, Q. Wang and Y. X. Li, Energy Environ. Sci., 2011, 4, 3652.

18 A. L. Linsebigler, G. Q. Lu and J. T. Yates, Chem. Rev., 1995, 95, 735 .

19 T. Ohno, K. Tokieda, S. Higashida and M. Matsumura, Appl. Catal., A, 2003, 244, 383.
20 H. G. Yang, C. H. Sun, S. Z. Qiao, J. Zou, G. Liu, S. C. Smith, H. M. Cheng and G. Q. Lu, Nature, 2008, 453, 638.

21 N. Iordanova, M. Dupuis and K. M. Rosso, J. Chem. Phys., 2005, 122, 144305.

22 J. P. Perdew, J. A. Chevary, S. H. Vosko, K. A. Jackson, M. R. Pederson, D. J. Singh and C. Fiolhais, Phys. Rev. B: Condens. Matter, 1992, 46, 6671.

23 J. P. Perdew, K. Burke and Y. Wang, Phys. Rev. B: Condens. Matter, 1996, 54, 16533.

24 J. P. Perdew, K. Burke and M. Ernzerhof, Phys. Rev. Lett., 1996, 77, 3865.

25 A. D. Becke, J. Chem. Phys., 1993, 98, 5648.

26 H. J. Monkhorst and J. D. Pack, Phys. Rev. B: Solid State, 1976, 13, 5188.

27 M. Methfessel and A. T. Paxton, Phys. Rev. B, 1989, 40, 3616.

28 G. K. H. Madsen and D. J. Singh, Comput. Phys. Commun., 2006, 175, 67.

29 D. Alfè, Comput. Phys. Commun., 2009, 180, 2622.

30 N. S. Lewis and D. G. Nocera, Proc. Natl. Acad. Sci. U. S. A., 2006, 103, 15729.

31 J. H. Alstrum-Acevedo, M. K. Brennaman and T. J. Meyer, Inorg. Chem., 2005, 44, 6802.

32 Z. j. Han, F. Qiu, R. Eisenberg, P. L. Holland and T. D. Krauss, Science, 2012, 338, 1321.

33 G. Henkelman and H. Jónsson, J. Chem. Phys., 2000, 113, 9978.

34 G. Henkelman, B. P. Uberuaga and H. Jónsson, J. Chem. Phys., 2000, 113, 9901.

35 M. Oshikiri and F. Aryasetiawan, J. Phys. Soc. Jpn., 2000, 69, 2113.

36 M. Rohlfing, P. Krüger and J. Pollmann, Phys. Rev. B: Condens. Matter, 1998, 57, 6485.

37 J. Heyd, J. E. Peralta, G. E. Scuseria and R. L. Martin, J. Chem. Phys., 2005, 123, 174101.

38 J. C. Wu, J. W. Zheng, C. L. Zacherl, P. Wu, Z. K. Liu and R. Xu, J. Phys. Chem. C, 2011, 115, 19741.

39 M. Shishkin and G. Kresse, Phys. Rev. B: Condens. Matter Mater. Phys., 2007, 75, 235102.

40 M. Kh. Rabadanov, Kristallografiya, 1995, 40, 21.

41 E. H. Kisi and M. M. Elcombe, Acta Crystallogr., Sect. C: Cryst. Struct. Commun., 1989, 45, 1867.

42 Y. P. Hong, Z. Lin, J. Huang, Y. J. Wang and F. Huang, Nanoscale, 2011, 3, 1512.

43 M. Pastore and F. De Angelis, ACS Nano, 2010, 4, 556. 